\title{
Nonlinear Graphene Nanoplasmonics
}

\author{
Joel D. Cox ${ }^{*, \dagger,+}$ and F. Javier García de Abajo* ${ }^{*, \S}$ \\ $\dagger$ Center for Nano Optics, University of Southern Denmark, Campusvej 55, DK-5230 \\ Odense M, Denmark \\ $\ddagger$ Danish Institute for Advanced Study, University of Southern Denmark, Campusvej 55, \\ DK-5230 Odense M, Denmark \\ \ICFO-Institut de Ciencies Fotoniques, The Barcelona Institute of Science and \\ Technology, 08860 Castelldefels (Barcelona), Spain \\ §ICREA-Institució Catalana de Recerca i Estudis Avançats, Passeig Lluís Companys 23, \\ 08010 Barcelona, Spain
}

E-mail: cox@mci.sdu.dk; javier.garciadeabajo@nanophotonics.es

\section{Conspectus}

Nonlinear optics is limited by the weak nonlinear response of available materials, a problem that is generally circumvented by relying on macroscopic structures in which light propagates over many optical cycles, thus giving rise to accumulated unity-order nonlinear effects. While this strategy cannot be extended to subwavelength optics, such as in nanophotonic structures, one can alternatively use localized optical resonances with high quality factors to increase light-matter interaction times at the expense of inelastic losses partly associated with the nonlinear response. Plasmons -the collective oscillations of electrons in conducting media- offer the means to concentrate light into nanometric volumes, well below the light-wavelength-scale limit imposed by diffraction, amplifying the electromagnetic fields upon which nonlinear optical phenomena depend. Due to their abundant supply of free electrons, noble metals are the traditional material platform for plasmonics, and have thus dominated research in nanophotonics over the past several decades, despite exhibiting large ohmic losses and inherent difficulties to actively modulate plasmon resonances, which are primarily determined by composition and morphology.

Highly doped graphene has recently emerged as an appealing platform for plasmonics due to its unique optoelectronic properties, which give rise to relatively long-lived, highly confined, and actively tunable plasmon resonances that mainly appear in the infrared and terahertz frequency regimes. Efforts to extend graphene plasmonics to the near-infrared and visible ranges involve patterning of graphene into nanostructured elements, facilitating the optical excitation of localized resonances that can be blue-shifted through geometrical confinement while maintaining electrical tunabiliy. Besides these appealing plasmonic attributes, the conical electronic dispersion relation of graphene renders its charge carrier motion in response to light intrinsically anharmonic, resulting in a comparatively intense nonlinear optical response. The combined synergy of extreme plasmonic field enhancement and large intrinsic optical nonlinearity are now motivating intensive research efforts in nonlinear graphene plasmonics, the recent progress of which we discuss in this Account. We start with a description of the appealing properties of plasmons in graphene nanostructures down to molecular sizes, followed by a discussion of the unprecedented level of intrinsic optical nonlinearity in graphene, its enhancement by resonant coupling to its highly confined plasmons to yield intense high harmonic generation and Kerr non- 
linearities, the extraordinary thermo-optical capabilities of this material enabling large nonlinear optical switching down to the single-photon level, and its strong interaction with quantum emitters.

\section{Introduction}

Research in nanophotonics is strongly rooted in the study of noble metal nanostructures supporting plasmons, ${ }^{1}$ collective free-electron oscillations that can focus light into atomic length scales for a plethora of applications ranging from biosensing ${ }^{2}$ to photochemistry ${ }^{3}$ and photovoltaics. ${ }^{4}$ Despite substantial progress in nanofabrication technologies, plasmons in conventional metals are still hindered by their large ohmic losses and the difficulty in actively tuning their properties, determined by the intrinsic optical response of the metal, the morphology of the structure, and the surrounding dielectric environment. ${ }^{5}$ Graphene has emerged as an appealing platform for plasmonics due to its unique optoelectronic properties. More precisely, (1) plasmons are supported in this material only when it is highly doped, and their frequencies can be actively modulated by varying the doping level (e.g., through the application of gating voltages $\left.{ }^{6-13}\right)$; (2) ohmic losses are extremely low in the plasmons supported by highquality graphene, particularly when encapsulated in hexagonal boron nitride ${ }^{14}$ and kept at cryogenic temperatures, ${ }^{15}$ with observed lifetimes in the picosecond timescale and associated quality factors in the triple-digit range; (3) graphene plasmons can be strongly confined with respect to the light wavelength, with reduction factors of their in-plane wavelengths reaching two orders of magnitude, and even higher levels of confinement when combined with metals to sustain acoustic modes. ${ }^{16,17}$

In its pristine form, graphene is a zero-gap semiconductor that exhibits $2.3 \%$ broadband light absorption associated with vertical interband optical transitions between valence and conduction Dirac cones. ${ }^{18,19}$ The addition of a doping charge carrier density $n$ shifts the Fermi energy to $E_{\mathrm{F}}=\hbar v_{\mathrm{F}} \sqrt{\pi n}$, where $v_{\mathrm{F}} \approx 10^{6} \mathrm{~m} / \mathrm{s}$ is the Fermi velocity, and produces a $2 E_{\mathrm{F}}$ interband absorption gap in which plasmon resonances emerge. In extended graphene samples, these excitations present an energy-momentum dispersion that lies far outside the free-space light cone, so that for a given optical frequency, there exists a large energy-momentum mismatch between the impinging light and the propagating plasmons that must be overcome in order to excite them. ${ }^{20}$ In practice, this imbalance can be compensated by the addition of external elements that provide extra momentum to the light, and most notably by the use of metallic tips, which have been instrumental in advancing the field of two-dimensional (2D) polaritonics. ${ }^{11,12,15,21-25}$

Patterning highly doped graphene into nanostructured elements facilitates the optical excitation of localized plasmon modes with resonance frequencies determined by the 2D geometry, providing the means to passively tune the plasmon resonance frequency range while maintaining its active electrical tunability. Indeed, plasmon resonance frequencies in a graphene nanostructure of characteristic size $D$ scale as $\omega_{\mathrm{p}} \propto \sqrt{E_{\mathrm{F}} / D}$, with typical experimentally observed Fermi energies reaching $\lesssim 1 \mathrm{eV}$ through electrostatic gating. ${ }^{9}$ These ideas were initially confirmed by pioneering spectroscopic measurements of electrically tunable $\mathrm{THz}$ plasmons in microstructured graphene ribbons, ${ }^{10,26,27}$ followed shortly thereafter by mid-IR plasmons in structures with lateral sizes of $\sim$ hundreds of nanometers. In this spectral range, interaction with the $0.2 \mathrm{eV}$ intrinsic optical phonons was originally thought to be detrimental, ${ }^{28}$ but their effect was later observed to be rather localized in frequency. ${ }^{29}$ Interaction with phonons in surrounding materials has been also investigated and shown to produce strong avoided crossing dispersion. ${ }^{30,31}$ These advances in the mid-IR spectral range are however hard to extrapolate to the more technologically relevant visible and near-IR regimes, although several strategies have been suggested, ${ }^{32}$ which could benefit from experimental efforts to explore extreme doping ${ }^{33}$ and smaller structures. ${ }^{34}$

The conical electronic band structure of graphene endows the carbon monolayer with 
an intrinsically anharmonic response to external electromagnetic fields, particularly for intraband motion of charge carriers driven back and forth across the Dirac point, producing efficient optical harmonic generation. ${ }^{35}$ The collective charge oscillations of graphene plasmons, which enhance the in-plane electric field driving the optical response, are also associated with intraband electronic transitions. The synergetic combination of the large intrinsic optical nonlinearity in graphene and the strong local field enhancement provided by its plasmons are thus stimulating extensive research efforts in nonlinear graphene plasmonics, an emerging field intersecting the themes of graphene plasmonics and nonlinear optics. ${ }^{36-53}$

The plasmon frequency scaling $\omega_{\mathrm{p}} \propto \sqrt{E_{\mathrm{F}} / D}$ is a strong motivation to consider structures with small lateral dimension $D$, which we discuss in this review by showing the response of graphene nanoislands of increasingly small size down to the molecular range. A reduction in size has the additional benefit that lower doping is needed to reach a given optical frequency, and in turn a lower Fermi energy increases the nonlinear response by engaging electronic motion closer to the Dirac point, where the anharmonicity associated with the conical electronic bands becomes more relevant.

\section{Quantum effects in the optical response of nanos- tructured graphene}

Low-energy plasmons in graphene nanostructures at least several tens of nanometers in size are well described in a classical electrodynamic framework as thin films of vanishing thickness characterized by the local 2D optical conductivity of extended graphene in the Dirac cone approximation. ${ }^{54}$ In nanostructures with dimensions $\lesssim 10 \mathrm{~nm}$, quantum-mechanical (QM) finite-size effects in the electronic structure become relevant, so the plasmonic response diverges from the classical prediction. ${ }^{55-57}$

The optical behavior of graphene in the infrared (IR) spectral region is dominated by $\pi$ band dynamics and can be reliably described using a simple nearest-neighbors tight-binding (TB) model. In this QM approach, electronic states of a graphene nanoisland containing $N_{\mathrm{C}}$ carbon atoms are described through a TB Hamiltonian $H_{\mathrm{TB}}$ connecting nearest neighbors with a hopping energy of $2.8 \mathrm{eV}$ to obtain singleelectron states $|j\rangle$ with energies $\hbar \varepsilon_{j}$, which can be expanded in the basis set of spin-degenerate carbon out-of-plane 2 p orbitals $|l\rangle$ at the carbon sites $\mathbf{R}_{l}$ according to $|j\rangle=\sum_{l} a_{j l}|l\rangle$, with realvalued coefficients $a_{j l}$ weighing the amplitude of orbital $|l\rangle$ in state $|j\rangle$. The optical response is characterized by the induced dipole moment $\mathbf{p}^{\text {ind }}=\sum_{l} \rho_{l}^{\text {ind }} \mathbf{R}_{l}$, computed from the induced charge $\rho_{l}^{\text {ind }}=-2 e \rho_{l l}$ obtained by solving the single-electron density matrix equation of motion

$$
\frac{\partial \rho}{\partial t}=-\frac{\mathrm{i}}{\hbar}\left[H_{\mathrm{TB}}-e \phi, \rho\right]-\frac{1}{\tau}\left(\rho-\rho^{0}\right),
$$

where $\phi_{l}=\phi_{l}^{\text {ext }}+\sum_{l^{\prime}} v_{l l^{\prime}} \rho_{l^{\prime}}^{\text {ind }}$ is the selfconsistent electrostatic potential, with the first and second terms accounting for external and induced contributions, respectively, and $v_{l l^{\prime}}$ denoting the Coulomb interaction between electrons in $2 \mathrm{p}$ carbon orbitals at $\mathbf{R}_{l}$ and $\mathbf{R}_{l^{\prime}}$. In particular, for irradiation with light of large wavelength compared with the size of the graphene structure, the external field amplitude $\mathbf{E}^{\text {ext }}$ is approximately uniform and we can take $\phi_{l}^{\text {ext }}=-\mathbf{R}_{l} \cdot \mathbf{E}^{\text {ext }}$. Equation 1 incorporates a phenomenological time $\tau$ for inelastic relaxation bringing the system to the unperturbed state described by $\rho_{j j^{\prime}}^{0}=\delta_{j j^{\prime}} f_{j}$, where $f_{j}$ is the occupancy of state $j$ in the absence of any external perturbation.

In Fig. 1 we illustrate the divergence of the QM description from classical electrodynamics by simulating the absorption spectra of equilateral triangular nanographenes as their size decreases. Specifically, we plot the absorption normalized to the triangle area as a function of the Fermi energy and impinging light energy. Incidentally, we assume the edges of these structures to be passivated by hydrogen atoms, and further impose the same hopping between nearest neighbors for all carbon bonds in the structure, regardless of their locations. In Fig. 1a 
a classical model ${ }^{8}$ is employed to describe the plasmonic response of the graphene nanotriangle, in which clear signatures of the electronhole pair continuum (i.e., for $\hbar \omega>2 E_{\mathrm{F}}$ ) and low-energy dipolar plasmons (resonances of frequency scaling as $\left.\omega_{\mathrm{p}} \propto \sqrt{E_{\mathrm{F}}}\right)$ are observed. In Fig. 1b, the same graphene nanotriangle is studied in an atomistic QM approach, ${ }^{55}$ showing plasmons in excellent agreement with the classical model, but already exhibiting striking differences in the interband transition region that are actually signatures of electronhole pair transitions in the discretized electronic structure. As the nanotriangle size decreases, subsequent panels in the upper row (Fig. 1bd) show how the plasmon resonances also deviate from the $\propto \sqrt{E_{\mathrm{F}}}$ frequency dependence, eventually making discrete jumps as each electronic state becomes populated, and presenting a strong spectral feature at higher energies associated with the HOMO-LUMO interband transitions. In the lower row (Fig. 1eh), we move further toward the molecular plasmonics regime, where doped graphene nanoislands with few-nanometer lateral size are more akin to charged polycyclic aromatic hydrocarbons (PAHs) exhibiting large gaps in their electronic spectra and electrically tunable plasmon resonances at visible frequencies.

\subsection{Molecular plasmonics}

The reduction in the size of the graphene nanotriangles investigated in Fig. 1 eventually leads to PAH molecules. For example, the structure in Fig. 1h corresponds to triphenylene, which exhibits a wide band gap $\sim 4 \mathrm{eV}$ in its neutral form, but displays resonances in the visible at $\sim 2 \mathrm{eV}$ when doped with one electron or hole. These types of resonances in charged PAHs have been experimentally investigated in the past, ${ }^{58-60}$ but their relation to graphene plasmonics now allows us to trace their origin to that of plasmons in highly doped graphene. Remarkably, the simple nearest-neighbors hopping model predicts the correct behavior (emergence of visible resonances when ionized, and similar spectra for positive and negative doping) and approximate spectral position of these molecular plasmons, in agreement with more sophisticated quantum chemistry simulations. ${ }^{61}$ Figure 2a shows absorption spectra (top), calculated within time-dependent density functional theory (TDDFT), compared with optical measurements in electrolyte solution ${ }^{62}$ (bottom). Similar results are obtained for other PAHs, and in all cases the observed spectra display finer structure than predicted by $\mathrm{TB}+\mathrm{RPA}$ or TDDFT, which can be attributed to the excitation of atomic vibrations in the molecules and incorporated in the simulations within the Frank-Condon approximation, ${ }^{63}$ as shown in Fig. 2b.

Remarkably, upon examination of available experiments for PAHs ionized with one ${ }^{60,62,63}$ and two ${ }^{60}$ electrons, we conclude that the trends of plasmon redshift with increasing size and blue shift with increasing doping observed in larger graphene samples are maintained down to PAHs composed of $\sim 20$ carbon atoms or less (see Fig. 2b), in good qualitative agreement with the simple TB+RPA model. ${ }^{61}$

We remark that $\mathrm{TB}+\mathrm{RPA}$ simulations provide an excellent quantitative description that allows us to predict the optical response of large ionized PAHs beyond the current capabilities of TDDFT, and further relate the existence of molecular plasmons to the presence of a finite HOMO-LUMO gap, ${ }^{61}$ which in turn is only possible when the number of carbon atoms is the same in the two graphene sublattices. ${ }^{64}$

\section{Nonlinear nanographene plasmonics: Coherent in- teraction}

While the anharmonic optical response of extended graphene to light can be attributed to its linear electronic dispersion relation, the excellent nonlinear optical properties of the 2D carbon layer persist in its nanostructured form, as evidenced by rigorous atomistic simulations of small equilateral graphene nanotriangles doped with only a few electrons (see Fig. 3). These results are obtained by extending the formalism introduced in the previous section beyond 
linear response. More precisely, we expand the density matrix $\rho=\sum_{n=0}^{\infty} \sum_{s=-n}^{n} \rho^{n s} \mathrm{e}^{-\mathrm{i} s \omega t}$ as a sum of the contributions of different perturbation orders $n$ and harmonic frequencies $s \omega$ under impinging continuous-wave (cw) optical illumination at frequency $\omega$; inserting this ansatz into eq 1 , we write the diagonal density matrix elements as ${ }^{38}$

$$
\rho_{l l}^{n s}=\frac{-1}{2 e} \sum_{l^{\prime}} \chi_{l l^{\prime}}^{0, s} \phi_{l^{\prime}}^{n s}+\sum_{j j^{\prime}} a_{j l} a_{j^{\prime} l} \eta_{j j^{\prime}}^{n s},
$$

where

$$
\chi_{l l^{\prime}}^{0, s}=\frac{2 e^{2}}{\hbar} \sum_{j j^{\prime}}\left(f_{j^{\prime}}-f_{j}\right) \frac{a_{j l} a_{j^{\prime} l} a_{j l^{\prime}} a_{j^{\prime} l^{\prime}}}{s \omega+\mathrm{i} / 2 \tau-\left(\varepsilon_{j}-\varepsilon_{j^{\prime}}\right)}
$$

is the noninteracting RPA susceptibility at frequency $s \omega$,

$$
\begin{aligned}
\eta_{j j^{\prime}}^{n s}=-\frac{e}{\hbar} \sum_{n^{\prime}=1}^{n-1} \sum_{s^{\prime}=-n^{\prime}}^{n^{\prime}} \sum_{l l^{\prime}} \frac{\left(\phi_{l}^{n^{\prime} s^{\prime}}-\phi_{l^{\prime}}^{n^{\prime} s^{\prime}}\right) a_{j l} a_{j^{\prime} l^{\prime}}}{s \omega+\mathrm{i} / 2 \tau-\left(\varepsilon_{j}-\varepsilon_{j^{\prime}}\right)} \\
\times \rho_{l l^{\prime}}^{n-n^{\prime}, s-s^{\prime}},
\end{aligned}
$$

is a source term constructed from perturbation orders below $n$, and $\phi_{l}^{n s}=$ $\phi_{l}^{\text {ext }} \delta_{n, 1}\left(\delta_{s,-1}+\delta_{s, 1}\right)-2 e \sum_{l^{\prime}} v_{l l^{\prime}} \rho_{l^{\prime} l^{\prime}}^{n s}$ is the contribution to the harmonic $s$ of the total potential at order $n$, with the external potential contributing only at order $n=1$. By numerically solving eq 2 , the nonlinear polarizabilities are calculated from the obtained time-dependent coefficients $\rho_{l l}^{n s}$; in particular, for harmonic fields along the impinging field polarization direction $\hat{\mathbf{e}}$, we have $\alpha_{s \omega}^{(n)}=-2 e\left(E^{\mathrm{ext}}\right)^{-n} \sum_{l} \rho_{l l}^{n s} \mathbf{R}_{l} \cdot \hat{\mathbf{e}}$. This procedure, which involves a computational effort that scales linearly with the perturbation order, produces results in excellent agreement with the direct numerical integration of eq 1 in the time domain, ${ }^{38}$ from which different harmonics are extracted by Fourier analysis.

The results of Fig. 3 indicate that, besides producing large shifts in the absorption spectrum, each additional doping electron can provide order-of-magnitude enhancements in harmonic generation and optical Kerr nonlinearity. Although graphene possesses a centrosymmetric crystal lattice, an even-ordered response is enabled in a finite nanostructure along directions in which the geometry breaks inversion symmetry. The resonance features in the linear response of Fig. 3a correlate with dominant peaks in the polarizabilities associated with second-harmonic generation (SHG, Fig. 3b), third-harmonic generation (THG, Fig. 3c), and the Kerr nonlinearity (Fig. 3d), both at the fundamental and/or generated frequencies, potentially leading to further enhancement when plasmonic resonances are simultaneously present at $\omega$ and $s \omega$, where $s$ is the harmonic index. This double-resonance condition can be met through geometrical and/or electrostatic tuning of localized plasmons, ${ }^{43}$ and is even more easily achievable in a wave-mixing configuration involving non-degenerate impinging fields that can independently target multiple resonance features. ${ }^{40}$

The perturbative treatment of the graphene optical response is valid strictly in the limit of vanishing impinging light intensity, which is easily surpassed in actual experiments probing nonlinear optical phenomena. The linear and nonlinear spectral lineshapes display an intensity dependence under $c w$ excitation above $\sim 1 \mathrm{GW} / \mathrm{m}^{2}$ intensity, as shown in time-domain simulations in Fig. 3e-g, obtained from the Fourier transform of the induced dipole over an optical cycle after reaching a steady-state regime. Inspection of the third-order perturbative Kerr nonlinearity, which contributes to the response oscillating at frequency $\omega$, gives rise to spectral shifts and broadening in the absorption cross section associated with the real and imaginary parts of $\alpha_{\omega}^{(3)}$, respectively, as shown in Fig. 3h; these modulations are amplified in the SHG and THG polarizabilities, which depend on higher powers of the plasmonic near-field. Eventually, as the impinging light intensity becomes sufficiently large, the anharmonicity associated with the Kerr nonlinearity can trigger a bistable optical response. ${ }^{38,42}$ 


\section{Extreme nonlinear opti- cal phenomena}

\subsection{Coherent nonperturbative regime}

Extreme nonlinear optical phenomena arise when an electronic system in an equilibrium state is illuminated by an optical field with sufficiently high intensity to appreciably populate excited states, so that the induced out-ofequilibrium electronic distribution and resulting optical response are ill-described by perturbation theory. High-harmonic generation (HHG) constitutes a well-known example of such an extreme process, and is well understood for individual atoms in terms of the so-called three-step model, in which an intense optical field triggers tunneling ionization of an electron from its parent ion, accelerates the electron away, and then re-collides it with the ion, emitting coherent radiation at very high harmonic orders of the impinging field. ${ }^{65,66}$ Recent experimental efforts have demonstrated an analogous process in crystalline media, where features in the electronic band structure and rich interplay of interband and intraband dynamics are imprinted in the HHG signal. ${ }^{67}$ In graphene, intraband dynamics within a Dirac cone is characterized by the dispersion relation $\varepsilon_{\mathbf{k}}=$ $v_{\mathrm{F}}|\mathbf{k}|$, with $\mathbf{k}$ denoting the electron wave vector, leading to a maximum surface current density $J \propto-e n v_{\mathrm{F}} \operatorname{sign}\{\sin (\omega t)\}$ under illumination by monochromatic light $E(t)=E_{0} \cos (\omega t)$ in the $\left|E_{0}\right| \rightarrow \infty$ limit; ${ }^{35}$ the current, proportional to the charge carrier density $n$, presents a squarewave temporal profile containing large contributions from odd-ordered harmonics in its Fourier decomposition. Although this simple estimate of the graphene nonlinear optical response neglects interband effects, which are predicted to impede this strong anharmonicity, ${ }^{68}$ the collective intraband motion associated with graphene plasmons and their ability to intensify the electric field within the graphene plane offer an appealing prescription to further enhance HHG.

The optical response of graphene nanotriangles under illumination by intense ultrashort light pulses is shown in Fig. 4 by mapping the emission intensity over a wide range of input pulse carrier frequencies, where at each input frequency the response is normalized to its respective maximium at the fundamental harmonic. For the undoped triangle presented in Fig. 4a, only odd-ordered harmonics of relatively low intensity are produced, despite the broken inversion symmetry in directions perpendicular to the equilateral triangle sides, while a striking increase in HHG from localized plasmons is clearly shown in Fig. $4 \mathrm{~b}$ at $E_{\mathrm{F}}=0.4 \mathrm{eV}$ doping, where the excitation frequency coincides with a prominent plasmon resonance.

\subsection{Incoherent thermally driven nonlinearities}

Saturable absorption (SA) is another nonperturbative nonlinear optical effect that consists of the quenching of optical absorption at high intensities, and is ubiquitous in photonic materials. Pristine graphene presents a broadband, electrically tunable $2.3 \%$ light absorption and remarkably low intensity threshold for $\mathrm{SA}$, and thus constitutes an appealing material platform for passive mode-locking lasers. ${ }^{69-71}$ The enhanced light-matter interaction associated with resonant excitation of graphene plasmons then produces an increase in light absorption that saturates at a low impinging light intensity threshold compared with other materials, an effect that can be customized by the electrostatic tunabiltiy of the carbon layer. In reality, the SA of graphene plasmons arises from an interplay between the strong coherent optical nonlinearity and incoherent nonlinear optical phenomena associated with the additional absorbed light energy that serves to dramatically elevate the electronic temperature in the carbon layer; previously absorbed photons undergo inelastic scattering, therefore producing a nonlinear effect that does not maintain optical phase coherence with subsequently impinging photons.

In Fig. 5a we schematically illustrate the excitation of plasmons in a highly doped graphene nanoribbon and the mechanism by which they 
dissipate: in a simple treatment, the plasmon decays directly to phonons in the carbon lattice at a phenomenological rate $\gamma^{0}$; in a more realistic scenario, the system relaxes at a rate $\gamma^{T}$ to a local equilibrium at an elevated electron temperature $T$ and shifted chemical potential $\mu$ (computed by enforcing conservation of electron number and energy), while electrons continuously transfer their energy to the lattice (i.e., by exciting phonons) at a rate $\gamma^{\mathrm{ph}}$. Results obtained from atomistic time-domain simulations of a graphene nanoribbon excited by pulses of fixed fluence and varying FWHM duration $\Delta$ are presented in Fig. 5b-e, illustrating the dramatic optically induced change in the instantaneous chemical potential and electron temperature that define the local equilibrium state. Comparison of these two descriptions indicates that their discrepancy becomes more apparent for pulses of longer duration, signifying the importance of the out-of-equilibrium electron dynamics associated with extreme nonlinear optical phenomena. ${ }^{45,52,72}$

\section{Applications and per- spectives}

The strong spatial confinement of graphene plasmons combined with the possibility of actively modulating the optical response of the material through optical gating and thermooptical heating makes it feasible to explore disruptive applications, three of which we discuss in this section.

\subsection{Strong coupling to quantum emitters}

The intense near-field enhancement associated with resonantly illuminated plasmonic nanostructures offers the means to interface optical fields with atomic systems, both natural (e.g., atoms and molecules) and artificial (e.g., quantum dots), thereby controlling light-matter interactions at the quantum level. Metal nanostructures have been widely studied in this context, where the strong coupling of plasmonic modes to long-lived electronic transitions in high-yield quantum emitters (QEs) facilitates optical sensing, single-photon generation, and coherent energy transfer on the nanoscale. ${ }^{73,74}$ In these applications, the limitations of noble metal plasmons, namely their large losses and lack of tunability, are circumvented by graphene plasmons, which are endowed with an optical nonlinearity by the fermionic nature of a proximal QE. In Fig. 6 we highlight several exciting predictions based on strong light-matter coupling of electrically tunable localized graphene plasmons in nanodisks with QEs, including strong coupling (Fig. 6a,b), large vacuum Rabi splittings (Fig. 6c), and optical nonlinearity associated with plasmon blockade in an emitternanodisk composite system. These theoretical predictions are yet waiting to be realized in experiment, in part due to a lack of good quantum light sources, with long-lived electronic transitions and high quantum efficiency, operating in the mid-IR and terahertz frequency ranges.

Plasmon-enhanced harmonic generation constitutes a possible mechanism to bridge the energy mismatch between electrically tunable IR graphene plasmons and a near-IR excitation in a neighboring QE. We explore this concept in Fig. 6e-j for a hybrid system consisting of a graphene nanodisk with diameter $D=40 \mathrm{~nm}$ and a two-level QE placed a distance $d$ directly above its center. The $\mathrm{QE}$ is characterized by a dipole moment $\vec{\mu}_{12}$ associated with the $|1\rangle \leftrightarrow|2\rangle$ transition of energy $\hbar \varepsilon_{12}=3 \hbar \omega_{\mathrm{p}}$. Impinging light of frequency $\omega \approx \omega_{\mathrm{p}}$ can generate a substantial plasmon-assisted third-harmonic near-field that resonantly drives the QE (see Figs. 6e,f). To estimate the strength of the nonlinear atom-plasmon interaction, we consider the QE dipole moment to be oriented parallel to the graphene plane and of magnitude $1 e \times \mathrm{nm}$ ( 50 Debye, commensurate with quantum dot excitons $\left.{ }^{75}\right)$; we also choose a phenomenological QE relaxation rate $\Gamma_{0}=10^{9} \mathrm{~s}^{-1}$ from the excited state $|2\rangle$ to ground state $|1\rangle$. As shown in Fig. 6g,h, the effective Rabi frequency $\Omega$ driving the QE (due to the plasmon-mediated nonlinear field at frequency $3 \omega$ ) and its self interaction $G$ (arising from the linear response of the graphene to the induced QE dipole at frequency $3 \omega)$ can reach considerably larger values than 
the QE linewidth under moderately intense $c w$ illumination, a phenomenon that can excite appreciable population in the QE (Fig. 6i) and be actively tuned by modulating the graphene Fermi energy (Fig. 6j). Following a similar strategy, potential applications in sensing and quantum nanophotonics could be realized by coupling electrically tunable mid-IR graphene plasmons to near-IR transitions in molecules of biological interest, and also to produce singlephoton sources of high fidelity.

\section{$5.2 \quad$ Nonlinear optical sensing}

The strong sensitivity of the graphene response to the application of external $d c$ fields can be pushed to the limit when considering the fields due to elementary charges and molecular dipoles. This idea constitutes the basis of a proposed method to detect charged or polar analytes through the changes that they induce in the optical response of nanographenes. Specifically, they give rise to spectral shifts, but they can also trigger a nonlinear response. Changes in the nonlinear signal produced by the presence of analytes have been investigated as a potentially efficient way of detection using metallic structures. ${ }^{76,77}$ However, the extraordinary nonlinear and optoelectronic properties of graphene can push the sensitivity close to the single molecule level. In particular, secondharmonic generation (SHG), which is absent in centrosymmetric graphene structures, is however enabled in symmetric nanographenes in the presence of a charged or polar molecule that breaks the symmetry, ${ }^{47}$ so that the presence of the latter is unveiled by tracking the graphene plasmon-enhanced SHG signal (see Fig. 7). Chemical changes produced by the analytes on the nanographenes can also introduce changes in their optical response, in particular when using ligands to bind them together, although these effects should be prevented by passivating the nanographenes by means of an atomically thin spacer, such as atomic layers of hexagonal boron nitride.

\subsection{Single-plasmon optical switch}

The optical and thermal properties of graphene are governed by a comparatively smaller number of electrons than in noble metals, thus leading to extraordinary thermo-optical properties associated with ultrafast all-optical modulation. ${ }^{52,78-80}$ The low electronic heat capacity of graphene endowed by its linear dispersion relation results in dramatic elevations in the conduction electron temperature upon resonant excitation of graphene plasmons, reaching thousands of degrees ${ }^{81,82}$ and significantly modifying the optical response on picosecond timescales, between the moment after electrons have thermalized and their cooling to the lattice by exciting phonons. In particular, plasmon modes predicted to be enabled by such rise of electron temperature ${ }^{32,78}$ were eventually observed through the use of pump-probe IR nanoscopy. ${ }^{79}$ For high-quality graphene, electron-phonon coupling occurs at a low pace, ${ }^{83}$ suggesting the possibility of spatially patterning the temperature profile in a graphene sheet under $c w$ optical pumping to dynamically modify the plasmonic landscape. ${ }^{84}$

Recent realistic simulations by the authors also predict that the change in the optical response of small graphene islands when absorbing the energy of one plasmon and transforming it into electronic heat is sufficient to dramatically shift the absorption spectral features, thus supporting the possibility of realizing a single-plasmon optical switch, ${ }^{85}$ as illustrated in Fig. 8. Although this type of switch does not preserve optical coherence between pump and probe, it could effectively realize the important goal of achieving optical switching at the level of a single quantum (i.e., by using a single photon) in a solid-state environment.

\section{Concluding remarks}

The combination of large field confinement and enhancement produced by graphene plasmons, the strong nonlinear response in this atomically thin material, its extraordinary sensitivity to external $d c$ fields, and its low electronic heat capacity enable applications in important fron- 
tiers of nanophotonics, and in particular, in the achievement of unity-order nonlinear effects at the nanoscale. Besides the implications for improved nonlinear optics, this synergetic combination of extraordinary properties in graphene holds strong potential for quantum optics, light modulation, and optical sensing. We have reviewed some of these possibilities in light of recent realistic theoretical predictions. A major challenge in the implementation of these ideas is the spectral range in which plasmons have so far been observed in graphene, which is limited to wavelengths of a few microns and above (i.e., the mid-IR and $\mathrm{THz}$ domains). The extension to shorter wavelengths in the technologically appealing near-IR region constitutes a challenge for which a reduction in the lateral size of the structures appears to be a plausible solution, for example through the use of molecular self-assembly. ${ }^{86-88}$ For quantum optics applications, one could alternatively use mid-IR quantum emitters, such as minibands and defects in 2D semiconductors. Device integration is also hampered by poor plasmonphoton coupling due to large momentum mismatch, ${ }^{20}$ yet a plausible strategy would consist in exciting graphene plasmons with electrical input/output, so that an electrical signal (e.g., through inelastic electron tunneling ${ }^{89-92}$ ) could be used to create plasmons on demand at designated positions; these excitations could then be processed by a photon-free, purely plasmonic device, and ultimately an output signal be read also through electrical means. These possibilities in combination with the extraordinary nonlinear optical capabilities of graphene constitute a promising platform for future disruptive nanophotonic applications and devices.

\section{Authors' biographies}

Joel D. Cox is a D-IAS Assistant Professor with the Center for Nano Optics in the Mads Clausen Institute at the University of Southern Denmark. His research expertise pertains to nanophotonics, nonlinear and quantum optics, and condensed matter physics.

F. Javier García de Abajo is an ICREA Re- search Professor at ICFO, where he leads the Nanophotonics Theory Group. His interests include surface science, nanophotonics, and electron microscopy.

\section{Acknowledgments}

This work has been supported in part by the Spanish MINECO (MAT2017-88492-R and SEV2015-0522), the European Research Council (Advanced Grant 789104-eNANO), the European Commission (Graphene Flagship 785219), the Catalan CERCA Program, and Fundació Privada Cellex. The Center for Nano Optics is financially supported by the University of Southern Denmark (SDU 2020 funding). J.D.C was supported by VILLUM Fonden (Grant No. 16498).

\section{References}

1 Born, M.; Wolf, E. Principles of Optics: Electromagnetic Theory of Propagation, Interference and Diffraction of Light; Cambridge University Press: Cambridge, 1999.

2 Brolo, A. G. Plasmonics for future biosensors. Nat. Photon. 2012, 6, 709-713.

3 Baffou, G.; Quidant, R. Nanoplasmonics for Chemistry. Chem. Soc. Rev. 2014, 38983907.

4 Clavero, C. Plasmon-Induced Hot-Electron Generation at Nanoparticle/Metal-Oxide Interfaces for Photovoltaic and Photocatalytic Devices. Nat. Photon. 2014, 8, 95103.

5 Boriskina, S. V.; Cooper, T. A.; Zeng, L.; Ni, G.; Tong, J. K.; Tsurimaki, Y.; Huang, Y.; Meroueh, L.; Mahan, G.; Chen, G. Losses in plasmonics: from mitigating energy dissipation to embracing lossenabled functionalities. Adv. Opt. Photon. 2017, 9, 775-826.

6 Jablan, M.; Buljan, H.; Soljačić, M. Plasmonics in graphene at infrared frequencies. Phys. Rev. B 2009, 80, 245435. 
7 Vakil, A.; Engheta, N. Transformation optics using graphene. Science 2011, 332, 1291-1294.

8 Koppens, F. H. L.; Chang, D. E.; García de Abajo, F. J. Graphene Plasmonics: A Platform for Strong Light-Matter Interactions. Nano Lett. 2011, 11, 3370-3377.

9 Chen, C. F.; Park, C. H.; Boudouris, B. W.; Horng, J.; Geng, B.; Girit, C.; Zettl, A.; Crommie, M. F.; Segalman, R. A.; Louie, S. G.; Wang, F. Controlling Inelastic Light Scattering Quantum Pathways in Graphene. Nature 2011, 471, 617-620.

10 Ju, L.; Geng, B.; Horng, J.; Girit, C.; Martin, M.; Hao, Z.; Bechtel, H. A.; Liang, X.; Zettl, A.; Shen, Y. R.; Wang, F. Graphene plasmonics for tunable terahertz metamaterials. Nat. Nanotech. 2011, 6, 630-634.

11 Fei, Z.; Rodin, A. S.; Andreev, G. O.; Bao, W.; McLeod, A. S.; Wagner, M.; Zhang, L. M.; Zhao, Z.; Thiemens, M.; Dominguez, G.; Fogler, M. M.; Neto, A. H. C.; Lau, C. N.; Keilmann, F.; Basov, D. N. Gate-Tuning of Graphene Plasmons Revealed by Infrared NanoImaging. Nature 2012, 487, 82-85.

12 Chen, J.; Badioli, M.; Alonso-González, P.; Thongrattanasiri, S.; Huth, F.; Osmond, J.; Spasenović, M.; Centeno, A.; Pesquera, A.; Godignon, P.; Zurutuza Elorza, A.; Camara, N.; García de Abajo, F. J.; Hillenbrand, R.; Koppens, F. H. L. Optical NanoImaging of Gate-Tunable Graphene Plasmons. Nature 2012, 487, 77-81.

13 Grigorenko, A. N.; Polini, M.; Novoselov, K. S. Graphene Plasmonics. Nat. Photon. 2012, 6, 749-758.

14 Woessner, A.; Lundeberg, M. B.; Gao, Y.; Principi, A.; Alonso-González, P.; Carrega, M.; Watanabe, K.; Taniguchi, T.; Vignale, G.; Polini, M.; Hone, J.; Hillenbrand, R.; Koppens, F. H. Highly Confined Low-Loss Plasmons in Graphene-Boron Nitride Heterostructures. Nat. Mater. 2015, 14, 421-425.
15 Ni, G. X.; McLeod, A. S.; Sun, Z.; Wang, L.; Xiong, L.; Post, K. W.; Sunku, S. S.; Jiang, B.-Y.; Hone, J.; Dean, C. R.; Fogler, M. M.; Basov, D. N. Fundamental limits to graphene plasmonics. Nature 2018, $557,530-533$.

16 Lundeberg, M. B.; Gao, Y.; Asgari, R.; Tan, C.; Duppen, B. V.; Autore, M.; Alonso-González, P.; Woessner, A.; Watanabe, K.; Taniguchi, T.; Hillenbrand, R.; Hone, J.; Polini, M.; Koppens, F. H. L. Tuning quantum nonlocal effects in graphene plasmonics. Science 2017, 89, 035004.

17 Alcaraz Iranzo, D.; Nanot, S.; Dias, E. J. C.; Epstein, I.; Peng, C.; Efetov, D. K.; Lundeberg, M. B.; Parret, R.; Osmond, J.; Hong, J.-Y.; Kong, J.; Englund, D. R.; Peres, N. M. R.; Koppens, F. H. L. Probing the ultimate plasmon confinement limits with a van der Waals heterostructure. Science 2018, 360, 291-295.

18 Nair, R. R.; Blake, P.; Grigorenko, A. N.; Novoselov, K. S.; Booth, T. J.; Stauber, T.; Peres, N. M. R.; Geim, A. K. Fine structure constant defines visual transparency of graphene. Science 2008, 320, 1308.

19 Mak, K. F.; Sfeir, M. Y.; Wu, Y.; Lui, C. H.; Misewich, J. A.; Heinz, T. F. Measurement of the optical conductivity of graphene. Phys. Rev. Lett. 2008, 101, 196405.

20 Dias, E. J. C.; García de Abajo, F. J. Fundamental Limits to the Coupling between Light and 2D Polaritons. ACS Nano 2019, 13, 5184-5197.

21 Hillenbrand, R.; Taubner, T.; Keilmann, F. Phonon-enhanced light-matter interaction at the nanometer scale. Nature 2002, 418, $159-162$.

22 Low, T.; Chaves, A.; Caldwell, J. D.; Kumar, A.; Fang, N. X.; Avouris, P.; Heinz, T. F.; Guinea, F.; MartinMoreno, L.; Koppens, F. Polaritons in layered two-dimensional materials. Nat. Mater. 2017, 16, 182. 
23 Basov, D. N.; Fogler, M. M.; García de Abajo, F. J. Polaritons in van der Waals Materials. Science 2016, 354, aag1992.

24 Nikitin, A. Y.; Alonso-González, P.; Vélez, S.; Mastel1, S.; Centeno, A.; Pesquera, A.; Zurutuza, A.; Casanova, F.; Hueso, L. E.; Koppens, F. H. L.; Hillenbrand, R. Real-space mapping of tailored sheet and edge plasmons in graphene nanoresonators. Nat. Photon. 2016, 10, 239-243.

25 Li, P.; Dolado, I.; Alfaro-Mozaz, F. J.; Casanova, F.; Hueso, L. E.; Liu, S.; Edgar, J. H.; Nikitin, A. Y.; Vélez, S.; Hillenbrand, R. Infrared hyperbolic metasurface based on nanostructured van der Waals materials. Science 2018, 359, 892-896.

26 Yan, H.; Li, X.; Chandra, B.; Tulevski, G.; Wu, Y.; Freitag, M.; Zhu, W.; Avouris, P.; Xia, F. Tunable infrared plasmonic devices using graphene/insulator stacks. Nat. Nanotech. 2012, 7, 330-334.

27 Strait, J. H.; Nene, P.; Chan, W.-M.; Manolatou, C.; Tiwari, S.; Rana, F.; Kevek, J. W.; McEuen, P. L. Confined plasmons in graphene microstructures: Experiments and theory. Phys. Rev. B 2013, 87, $241410(\mathrm{R})$.

28 Yan, H.; Low, T.; Zhu, W.; Wu, Y.; Freitag, M.; Li, X.; Guinea, F.; Avouris, P.; Xia, F. Damping pathways of mid-infrared plasmons in graphene nanostructures. Nat. Photon. 2013, 7, 394-399.

29 Bezares, F. J.; Saavedra, A. D. J. R. M.; Woessner, A.; Alonso-González, P.; Amenabar, I.; Chen, J.; Bointon, T. H.; Dai, S.; Fogler, M. M.; Basov, D. N.; Hillenbrand, R.; Craciun, M. F.; García de Abajo, F. J.; Russo, S.; Koppens, F. H. L. Intrinsic plasmon-phonon interactions in highly doped graphene: A near-field imaging study. Nano Lett. 2017, 17, 59085913.

30 Brar, V. W.; Jang, M. S.; Sherrott, M.; Lopez, J. J.; Atwater, H. A. Highly
Confined Tunable Mid-Infrared Plasmonics in Graphene Nanoresonators. Nano Lett. 2013, 13, 2541-2547.

31 Brar, V. W.; Seok Jang, M.; Sherrott, M. C.; Kim, S.; Lopez, J. J.; Kim, L. B.; Choi, M.; Atwater, H. A. Hybrid Surface-Phonon-Plasmon Polariton Modes in Graphene/Monolayer h-BN Heterostructures. Nano Lett. 2014, 14, 3876-3880.

32 García de Abajo, F. J. Graphene Plasmonics: Challenges and Opportunities. ACS Photon. 2014, 1, 135-152.

33 Khrapach, I.; Withers, F.; Bointon, T. H.; Polyushkin, D. K.; Barnes, W. L.; Russo, S.; Craciun, M. F.; Monica, F. Novel highly conductive and transparent graphene-based conductors. Adv. Mater. 2012, 24, 28442849 .

34 Wang, Z.; Li, T.; Almdal, K.; Mortensen, N. A.; Xiao, S.; Ndoni, S. Experimental Demonstration of Graphene Plasmons Working Close to the nearInfrared Window. Opt. Lett. 2016, 41, 5345-5348.

35 Mikhailov, S. A. Non-linear electromagnetic response of graphene. Europhys. Lett. 2007, 79, 27002.

36 Mikhailov, S. A. Theory of the giant plasmon-enhanced second-harmonic generation in graphene and semiconductor twodimensional electron systems. Phys. Rev. B 2011, 84, 045432.

37 Gorbach, A. V. Nonlinear graphene plasmonics: Amplitude equation for surface plasmons. Phys. Rev. A 2013, 87, 013830.

38 Cox, J. D.; García de Abajo, F. J. Electrically tunable nonlinear plasmonics in graphene nanoislands. Nat. Commun. 2014, 5,5725 .

39 Yao, X.; Tokman, M.; Belyanin, A. Efficient nonlinear generation of $\mathrm{THz}$ plasmons in graphene and topological insulators. Phys. Rev. Lett. 2014, 112, 055501. 
40 Cox, J. D.; García de Abajo, F. J. Plasmonenhanced nonlinear wave mixing in nanostructured graphene. ACS Photon. 2015, 2, 306-312.

41 Jablan, M.; Chang, D. E. Multiplasmon absorption in graphene. Phys. Rev. Lett. 2015, $114,236801$.

42 Christensen, T.; Yan, W.; Jauho, A.P.; Wubs, M.; Mortensen, N. A. Kerr nonlinearity and plasmonic bistability in graphene nanoribbons. Phys. Rev. B 2015, 92, $121407(\mathrm{R})$.

43 Manzoni, M. T.; Silveiro, I.; García de Abajo, F. J.; Chang, D. E. Second-Order Quantum Nonlinear Optical Processes in Single Graphene Nanostructures and Arrays. New J. Phys. 2015, 17, 083031.

44 Constant, T. J.; Hornett, S. M.; Chang, D. E.; Hendry, E. All-optical generation of surface plasmons in graphene. Nat. Phys. 2016, 12, 124-127.

45 Jadidi, M. M.; König-Otto, J. C.; Winnerl, S.; Sushkov, A. B.; Drew, H. D.; Murphy, T. E.; Mittendorff, M. Nonlinear terahertz absorption of graphene plasmons. Nano Lett. 2016, 16, 2734-2738.

46 Cox, J. D.; Silveiro, I.; García de Abajo, F. J. Quantum Effects in the Nonlinear Response of Graphene Plasmons. ACS Nano 2016, 10, 1995-2003.

47 Yu, R.; Cox, J. D.; García de Abajo, F. J. Nonlinear Plasmonic Sensing with Nanographene. Phys. Rev. Lett. 2016, $117,123904$.

48 Cox, J. D.; Marini, A.; García de Abajo, F. J. Plasmon-Assisted HighHarmonic Generation in Graphene. Nat. Commun. 2017, 8, 14380.

49 Rostami, H.; Katsnelson, M. I.; Polini, M. Theory of plasmonic effects in nonlinear optics: The case of graphene. Phys. Rev. B 2017, 95, 035416.
50 Kundys, D.; Duppen, B. V.; Marshall, O. P.; Rodriguez, F.; Torre, I.; Tomadin, A.; Polini, M.; Grigorenko, A. N. Nonlinear light mixing by graphene plasmons. Nano Lett. 2018, 18, 282-287.

51 Cox, J. D.; Yu, R.; García de Abajo, F. J. Analytical description of the nonlinear plasmonic response in nanographene. Phys. Rev. B 2017, 96, 045442 .

52 Cox, J. D.; García de Abajo, F. J. Transient nonlinear plasmonics in nanostructured graphene. Optica 2018, 5, 429-433.

53 Cox, J. D.; García de Abajo, F. J. Nonlinear atom-plasmon interactions enabled by nanostructured graphene. Phys. Rev. Lett. 2018, 121, 257403.

54 Yu, R.; Cox, J. D.; Saavedra, J. R. M.; García de Abajo, F. J. Analytical modeling of graphene plasmons. ACS Photon. 2017, 4, 3106-3114.

55 Thongrattanasiri, S.; Manjavacas, A.; García de Abajo, F. J. Quantum finite-size effects in graphene plasmons. ACS Nano 2012, 6, 1766-1775.

56 Christensen, T.; Wang, W.; Jauho, A.P.; Wubs, M.; Mortensen, N. A. Classical and Quantum Plasmonics in Graphene Nanodisks: Role of Edge States. Phys. Rev. B 2014, 90, 241414(R).

57 Silveiro, I.; Plaza Ortega, J. M.; García de Abajo, F. J. Quantum nonlocal effects in individual and interacting graphene nanoribbons. Light Sci. Appl. 2015, 4, e241.

58 Balk, P.; Hoijtink, G. J.; Schreurs, J. W. H. Electronic spectra of mono- and di-negative aromatic ions. Rec. Trav. Chim. Pays Bas 1957, 76, 813-823.

59 Hoijtink, G. J. Correlations between the electronic spectra of alternant hydrocarbon molecules and their mono- and di-valent ions. Mol. Phys. 1959, 2, 85-95. 
60 Hoijtink, G. J.; Zandstra, P. J. Polarization of electronic transitions in aromatic hydorcarbon molecules and their mono- and divalent ions. Mol. Phys. 1960, 3, 371-389.

61 Manjavacas, A.; Marchesin, F.; Thongrattanasiri, S.; Koval, P.; Nordlander, P.; Sánchez-Portal, D.; García de Abajo, F. J. Tunable Molecular Plasmons in Polycyclic Aromatic Hydrocarbons. ACS Nano 2013, 7, 3635-3643.

62 Lauchner, A.; Schlather, A.; Manjavacas, A.; Cui, Y.; McClain, M. J.; Stec, G. J.; García de Abajo, F. J.; Nordlander, P.; Halas, N. J. Molecular Plasmonics. Nano Lett. 2015, 15, 6208-6214.

63 Cui, Y.; Lauchner, A.; Manjavacas, A.; García de Abajo, F. J.; Halas, N. J.; Nordlander, P. Molecular Plasmon-Phonon Coupling. Nano Lett. 2016, 16, 6390-6395.

64 Fernández-Rossier, J.; Palacios, J. J. Magnetism in graphene nanoislands. Phys. Rev. Lett. 2007, 99, 177204.

65 Corkum, P. B. Plasma perspective on strong-field multiphoton ionization. Phys. Rev. Lett. 1993, 71, 1994-1997.

66 Lewenstein, M.; Balcou, P.; Ivanov, M. Y.; L'Huillier, A.; Corkum, P. B. Theory of high-harmonic generation by low-frequency laser fields. Phys. Rev. A 1994, 49, 21172132.

67 Ghimire, S.; Reis, D. A. High-harmonic generation from solids. Nat. Phys. 2018, 15, 10-16.

68 Ishikawa, K. L. Nonlinear optical response of graphene in time domain. Phys. Rev. B 2010, 82, 201402(R).

69 Bao, Q.; Loh, K. P. Graphene Photonics, Plasmonics, and Broadband Optoelectronic Devices. ACS Nano 2012, 6, 3677-3694.

70 Marini, A.; Silveiro, I.; García de Abajo, F. J. Molecular Sensing with Tunable Graphene Plasmons. ACS Photon. 2015, 2, 876-882.
71 Marini, A.; Cox, J. D.; García de Abajo, F. J. Theory of Graphene Saturable Absorption. Phys. Rev. B 2017, 95, 125408.

72 Baudisch, M.; Marini, A.; Cox, J. D.; Zhu, T.; Silva, F.; Teichmann, S.; Massicotte, M.; Koppens, F. H. L.; Levitov, L. S.; García de Abajo, F. J.; Biegert, J. Analytical modeling of graphene plasmons. Nat. Commun. 2018, 9, 1018.

73 Tame, M. S.; McEnery, K. R.; Ş. K. Özdemir an J. Lee,; Maier, S. A.; Kim, M. S. Quantum plasmonics. Nat. Phys. 2013, 9, 329-340.

74 Bozhevolnyi, S. I.; Khurgin, J. B. The case for quantum plasmonics. Nat. Photon. 2017, 11, 398-400.

75 Stievater, T. H.; Li, X.; Steel, D. G.; Gammon, D.; Katzer, D. S.; Park, D.; Piermarocchi, C.; Sham, L. J. Rabi oscillations of excitons in single quantum dots. Phys. Rev. Lett. 2001, 87, 133603.

76 Neely, A.; Perry, C.; Varisli, B.; Singh, A. K.; Arbneshi, T.; Senapati, D.; Kalluri, J. R.; Ray, P. C. Ultrasensitive and highly selective detection of Alzheimer's disease biomarker using two-photon Rayleigh scattering properties of gold nanoparticle. ACS Nano 2009, 3, 2834-2840.

77 Mesch, M.; Metzger, B.; Hentschel, M.; Giessen, H. Nonlinear Plasmonic Sensing. Nano Lett. 2016, 16, 3155-3159.

78 Vafek, O. Thermoplasma polariton within scaling theory of single-layer graphene. Phys. Rev. Lett. 2006, 97, 266406.

79 Ni, G. X.; Wang, L.; Goldflam, M. D.; Wagner, M.; Fei, Z.; McLeod, A. S.; Liu, M. K.; Keilmann, F.; Özyilmaz, B.; Neto, A. H. C.; Hone, J.; Fogler, M. M.; Basov, D. N. Ultrafast Optical Switching of Infrared Plasmon Polaritons in High-Mobility Graphene. Nat. Photon. 2016, 10, 244-248.

80 Tan, S.; Argondizzo, A.; Wang, C.; Cui, X.; Petek, H. Ultrafast multiphoton thermionic 
photoemission from graphite. Phys. Rev. X 2017, 7, 011004.

81 Johannsen, J. C.; Ulstrup, S.; Cilento, F.; Crepaldi, A.; Zacchigna, M.; Cacho, C.; Turcu, I. C. E.; Springate, E.; Fromm, F.; Raidel, C.; Seyller, T.; Parmigiani, F.; Grioni, M.; Hofmann, P. Direct view of hot carrier dynamics in graphene. Phys. Rev. Lett. 2013, 111, 027403.

82 Gierz, I.; Petersen, J. C.; Mitrano, M.; Cacho, C.; Turcu, I. C. E.; Springate, E.; Stöhr, A.; Köhler, A.; Starke, U.; Cavalleri, A. Snapshots of non-equilibrium Dirac carrier distributions in graphene. Nat. Mater 2013, 12, 1119-1124.

83 Efetov, D. K.; Kim, P. Controlling electronphonon interactions in graphene at ultrahigh carrier densities. Phys. Rev. Lett. 2010, 105, 256805.

84 Yu, R.; Guo, Q.; Xia, F.; García de Abajo, F. J. Photothermal engineering of graphene plasmons. Phys. Rev. Lett. 2018, 121, 057404.

85 Cox, J. D.; García de Abajo, F. J. Single-Plasmon Thermo-Optical Switching in Graphene. Nano Lett. 2019, 19, DOI: 10.1021/acs.nanolett.9b00879.

86 Cai, J.; Ruffieux, P.; Jaafar, R.; Bieri, M.; Braun, T.; Blankenburg, S.; Muoth, M.; Seitsonen, A. P.; Saleh, M.; Feng, X.; Müllen, K.; Fasel, R. Atomically precise bottom-up fabrication of graphene nanoribbons. Nature 2010, 466, 470-473.

87 Ruffieux, P.; Wang, S.; Yang, B.; SánchezSánchez, C.; Liu, J.; Dienel, T.; Talirz, L.; Shinde, P.; Pignedoli, C. A.; Passerone, D.; Dumslaff, T.; Feng, X.; Müllen, K.; Fasel, R. On-surface synthesis of graphene nanoribbons with zigzag edge topology. Nature 2016, 531, 489.

88 Moreno, C.; Vilas-Varela, M.; Kretz, B.; Garcia-Lekue, A.; Costache, M. V.; Paradinas, M.; Panighel, M.; Ceballos, G.; Valenzuela, S. O.; Peña, D.; Mugarza, A. Bottom- up synthesis of multifunctional nanoporous graphene. Science 2018, 360, 199-203.

89 Walters, R. J.; van Loon, R. V. A.; Brunets, I.; Schmitz, J.; Polman, A. A Silicon-Based Electrical Source of Surface Plasmon Polaritons. Nat. Mater. 2010, 9, 21-25.

90 Zhang, M. C.; Allen, T. W.; DeCorby, R. G. Experimental study of optimized surfaceplasmon-mediated tunneling in metaldielectric multilayers. Appl. Phys. Lett. 2013, 103, 071109.

91 Svintsov, D.; Devizorova, Z.; Otsuji, T.; Ryzhii, V. Plasmons in tunnel-coupled graphene layers: Backward waves with quantum cascade gain. Phys. Rev. B 2016, 94, 115301.

92 de Vega, S.; García de Abajo, F. J. Plasmon Generation through Electron Tunneling in Graphene. ACS Photon. 2017, 4, 2367-2375.

93 Manjavacas, A.; Nordlander, P.; García de Abajo, F. J. Plasmon blockade in nanostructured graphene. ACS Nano 2012, 6, 17241731 . 

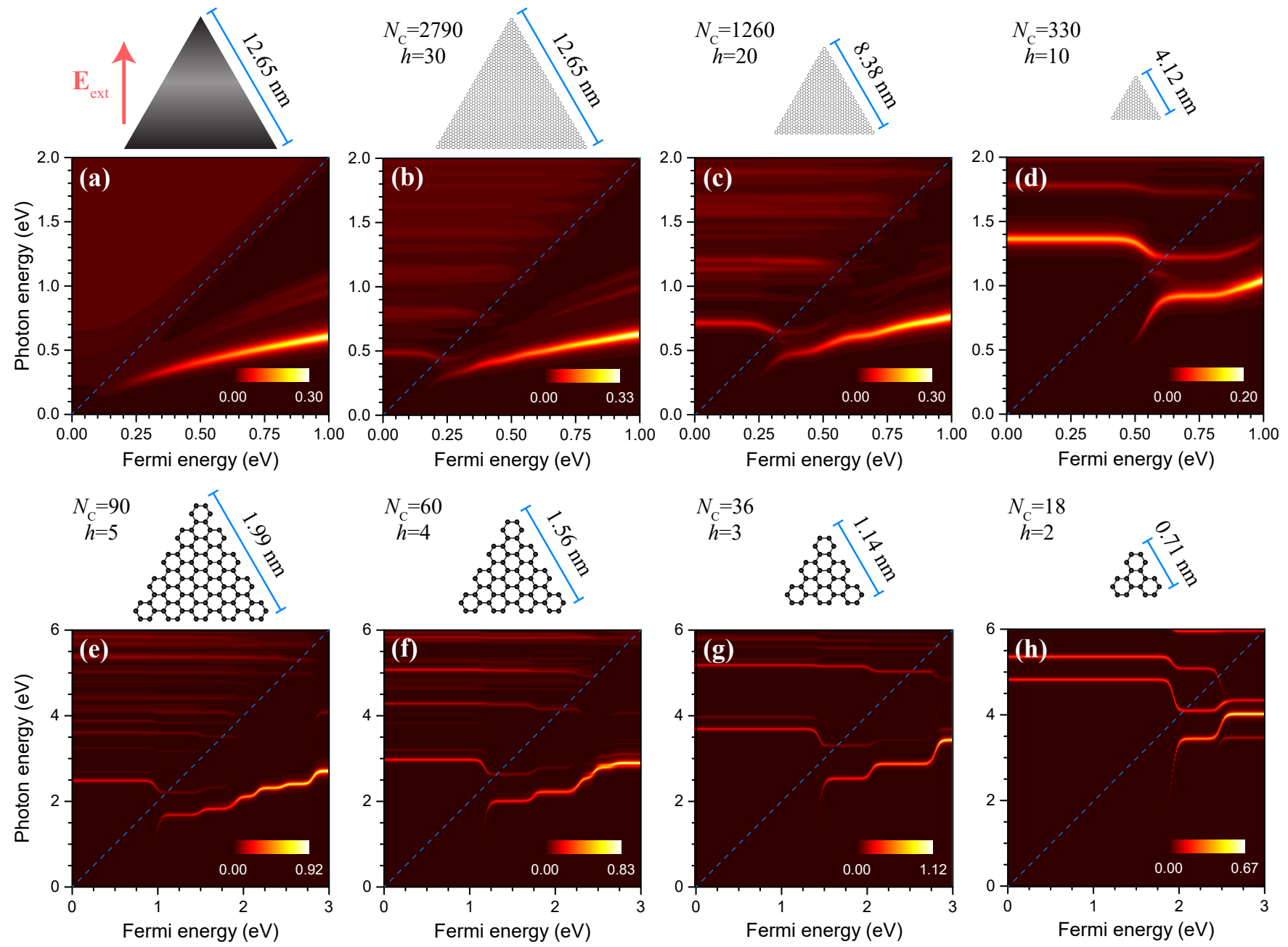

Figure 1: Transition between classical and quantum regimes in nanographene plasmons. In each panel we plot the absorption spectrum of the graphene nanotriangles illustrated above it as a function of Fermi energy $E_{\mathrm{F}}$ and incident photon energy $\hbar \omega$, with the interband threshold $\hbar \omega=2 E_{\mathrm{F}}$ marked by a dashed line and the color bar indicating the absorption cross section normalized to the triangle area. Panel (a) is obtained using a classical electrodynamic description, ${ }^{8}$ while the rest of the panels present quantum-mechanical (QM) simulations ${ }^{55}$ for triangles with entirely armchair edges. The graphene area is defined by multiplying the number of carbon atoms $N_{\mathrm{C}}$ by the graphene atomic density $n_{\mathrm{C}}=4 / 3 \sqrt{3} a \approx 3.8 \times 10^{15} \mathrm{~cm}^{-2}$, where $a=0.1421 \mathrm{~nm}$ is the $\mathrm{C}-\mathrm{C}$ bond distance. Classical theory (panel a) agrees well with $\mathrm{QM}$ simulations for the largest triangle under consideration (panel b, consisting of $N_{\mathrm{C}}=2790$ carbon atoms and $h=30$ benzene hexagons), and eventually deviates for increasingly small triangles (panels c-h). Light is considered to be incident along the carbon plane normal. The absorption cross section is independent of polarization due to the 3 -fold symmetry of the system. 

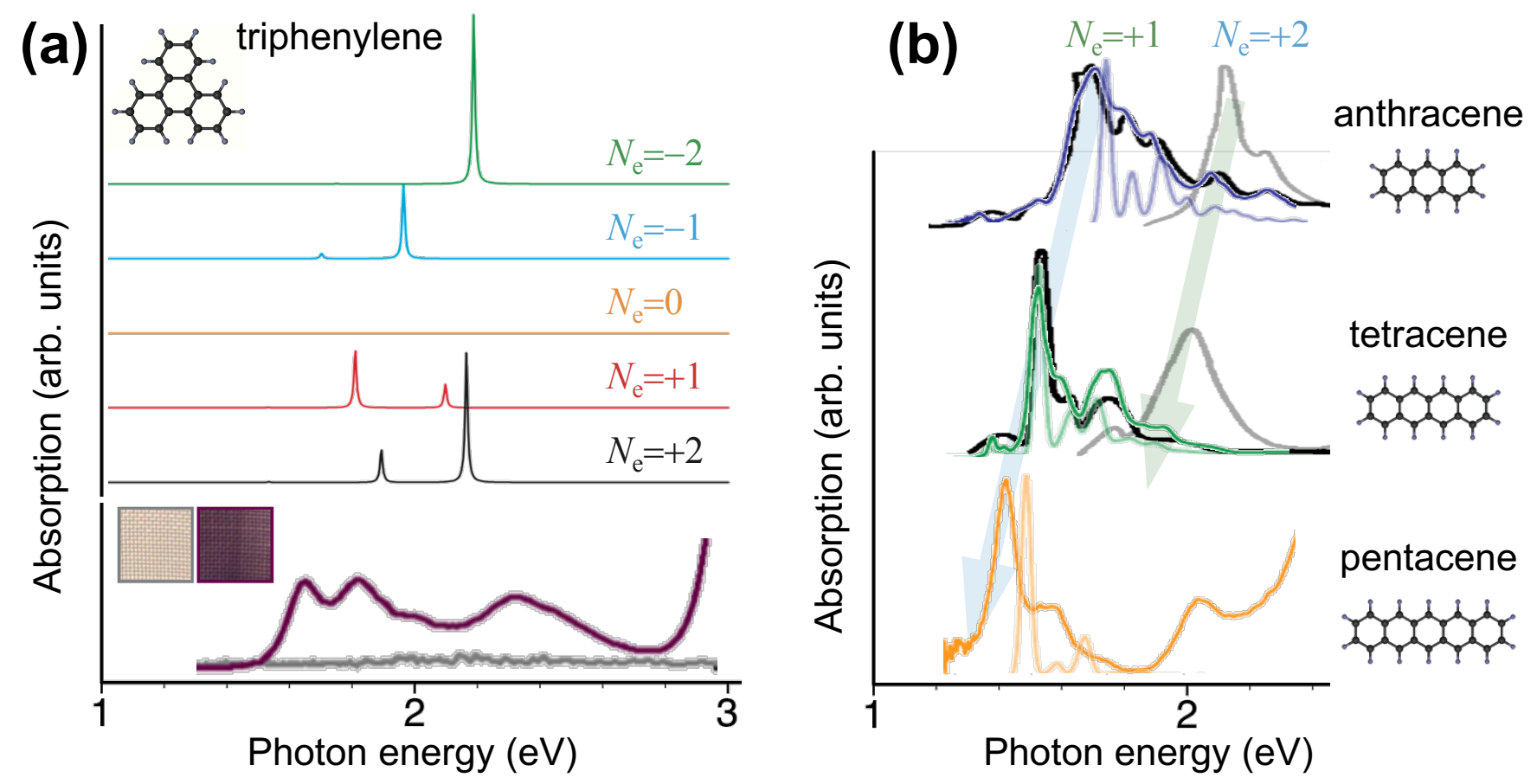

Figure 2: Molecular plasmons. (a) Top: TDDFT simulations of the absorption spectra of triphenylene when doped with $N_{\mathrm{e}}$ electrons $\left(N_{\mathrm{e}}<0\right.$ indicates hole doping). Adapted with permission from Ref. ${ }^{61}$ Copyright 2013 American Chemical Society. Bottom: measured optical absorption of neutral (gray curve) and singly reduced (purple curve) triphenylene in electrolyte solution; the inset shows photos of the electrode in the neutral (left) and reduced (right) states. Adapted with permission from Ref. ${ }^{62}$ Copyright 2015 American Chemical Society. (b) Absorption spectra of three acenes of increasing length in singly and doubly reduced states. Dark color curves: measurements from Ref. ${ }^{63}$ for $N_{\mathrm{e}}=1$. Light color curves: TDDFT simulations including coupling to atomic vibrations from Ref. ${ }^{63}$ for $N_{\mathrm{e}}=1$. Black $\left(N_{\mathrm{e}}=1\right)$ and gray $\left(N_{\mathrm{e}}=2\right)$ curves: measurements for anthracene and tetracene adapted with permission from Ref. ${ }^{60}$ Copyright 1960 Taylor \& Francis. 

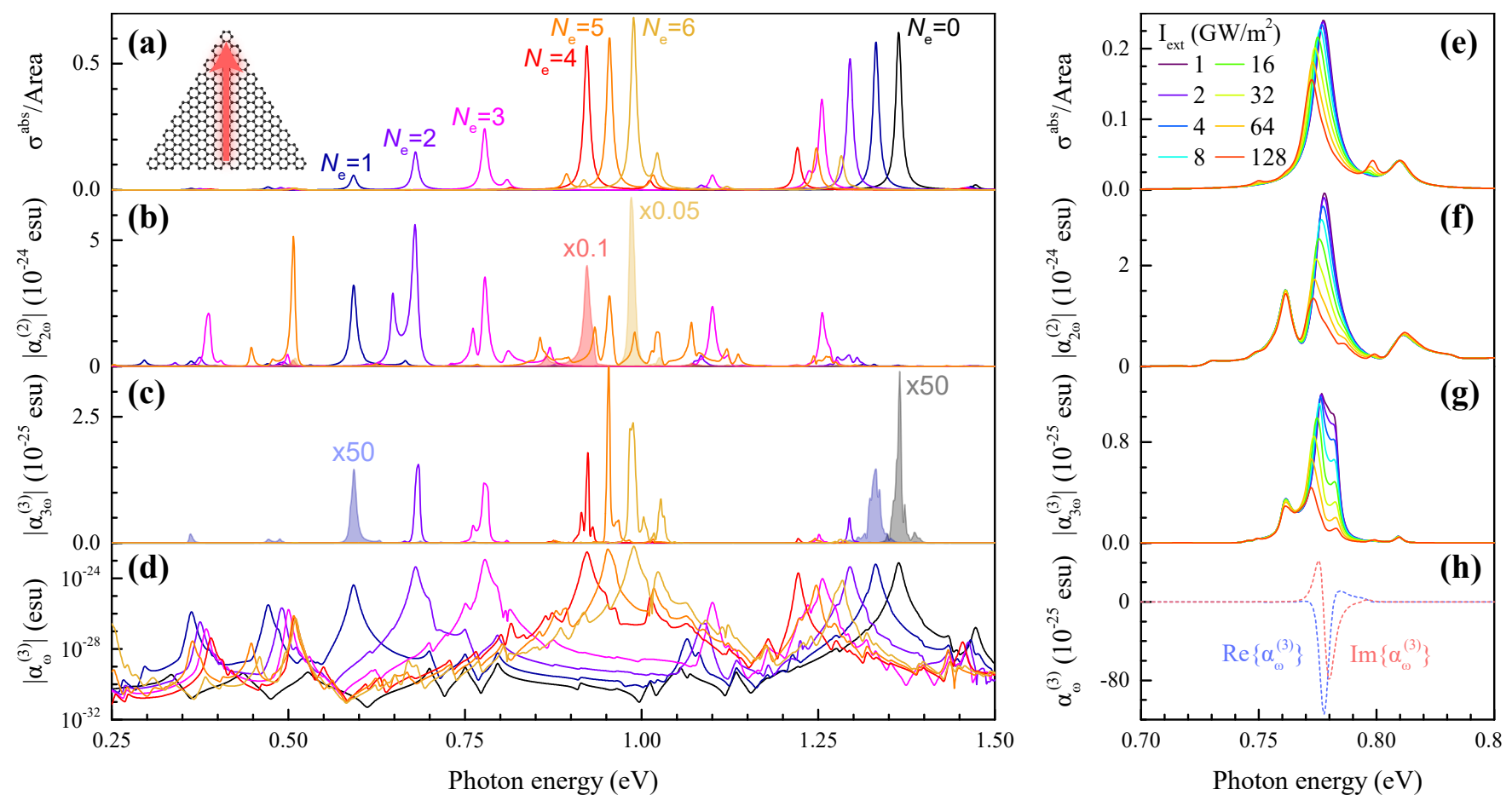

Figure 3: Nonlinear response in the near-perturbative regime. We consider an armchaired graphene nanotriangle consisting of $N_{\mathrm{C}}=330$ carbon atoms ( $4.12 \mathrm{~nm}$ side length) illuminated with external light of polarization as shown in the inset of (a) and doped with a finite number of electrons $N_{\mathrm{e}}$ (see labels). We plot the linear absorption cross-section (a), the SHG (b) and THG (c) polarizabilities, and the Kerr nonlinear polarizability (d), all calculated perturbatively within the TB+RPA approach. In (e-g) we show TB+RPA simulations for high $c w$ illumination intensities $\left(I_{\text {ext }}\right)$ at frequencies near the low-energy linear dipole plasmon under $N_{\mathrm{e}}=3$ doping, along with the real and imaginary parts of the perturbative Kerr polarizability in (h). Adapted with permission from Ref. ${ }^{38}$ Copyright 2014 Springer Nature. 

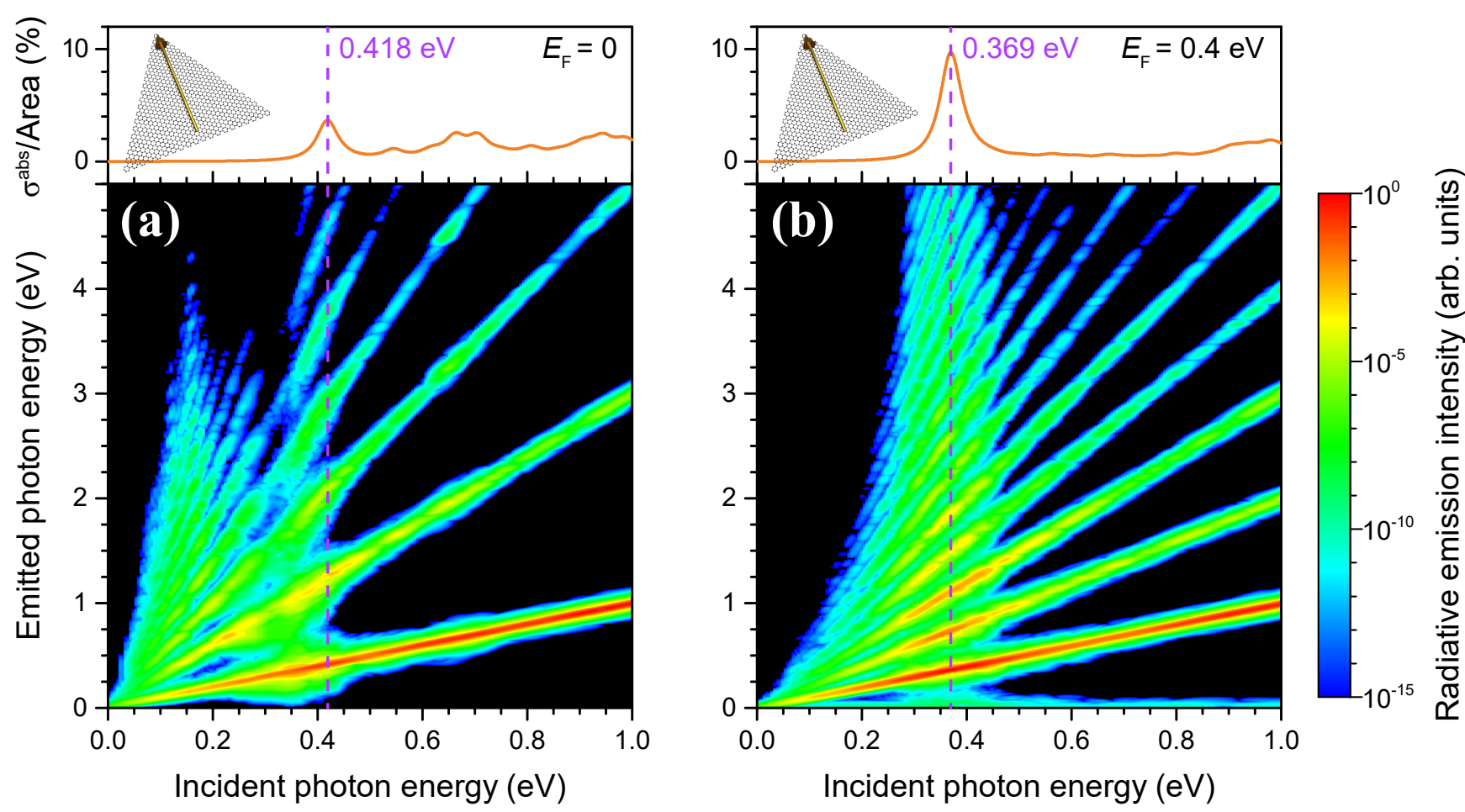

Figure 4: High-harmonic generation from nanographenes. We plot the light emission intensity arising from doped graphene nanotriangles with armchair edges and equal side lengths of $15 \mathrm{~nm}$ as a function of emitted photon energy (vertical axes) when we vary the incident photon energy (horizontal axes). These results are obtained within the time-domain TB+RPA approach using incident pulses of $100 \mathrm{fs}$ duration and $10^{12} \mathrm{~W} / \mathrm{m}^{2}$ peak intensity for undoped (a) and doped (b) graphene $(0.4 \mathrm{eV}$ Fermi energy). Both plots share the same color scale, normalized to the absolute maximum of (b). The top insets show the corresponding linear absorption spectra and the polarization of the incident light. Adapted with permission from Ref. ${ }^{48}$ Copyright 2017 Springer Nature. 

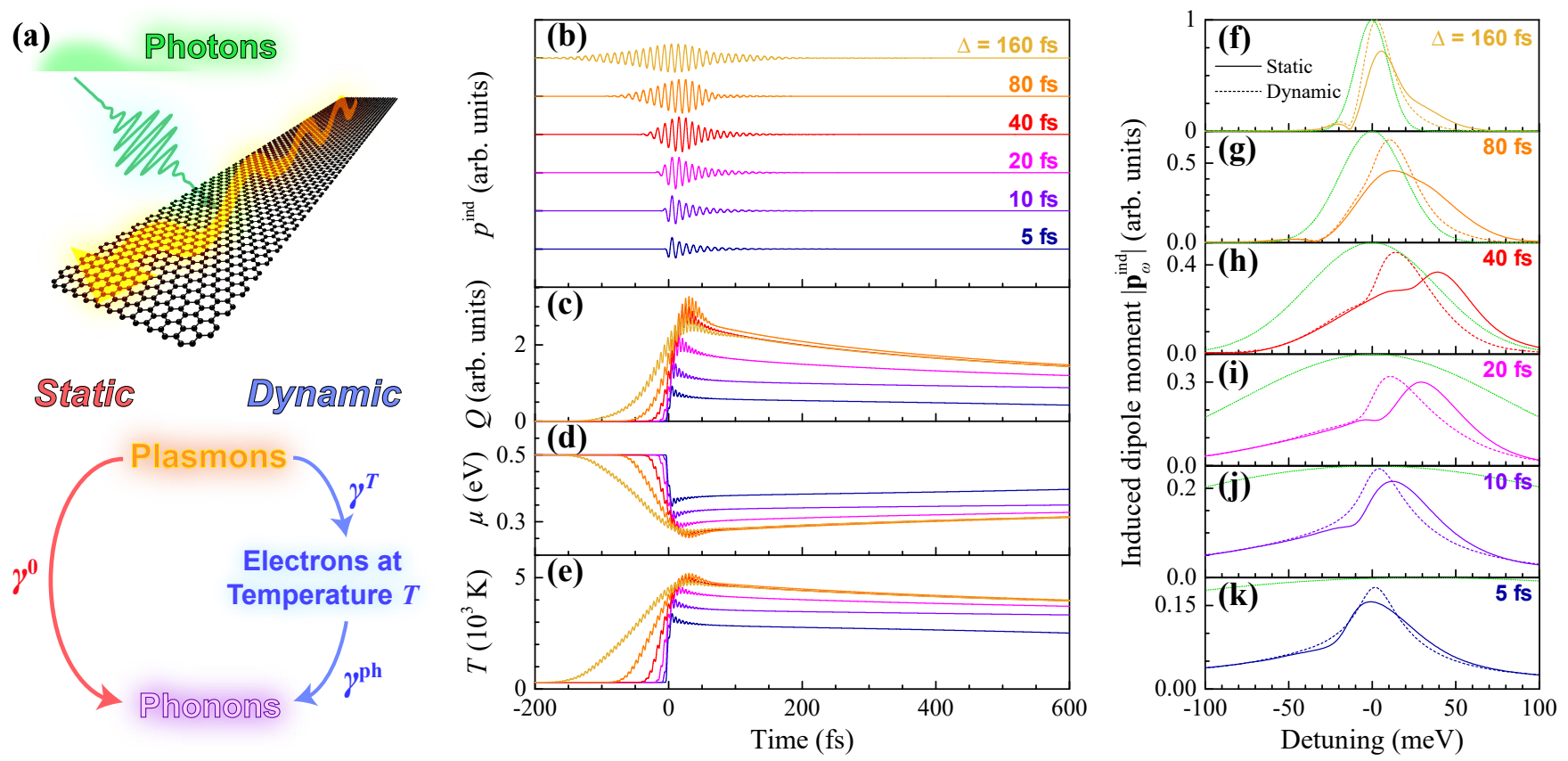

Figure 5: Plasmon-assisted ultrafast transient light absorption. (a) Plasmon decay in graphene is generally simulated through a phenomenological decay rate $\gamma^{0}$ (static model) that does not leave any trace of the plasmon energy on the response of the material. In a more realistic dynamical model (right), we consider the increase in electron temperature contributed by the dissipated plasmon energy at a rate $\gamma^{T}$, and the subsequent decay of electronic heat to phonons at a lower rate $\gamma^{\mathrm{ph}}$. (b-e) Temporal evolution of the induced polarization (b), electronic heat (c), chemical potential (d), and electron temperature (e) in a graphene ribbon (20 nm width, $0.5 \mathrm{eV}$ doping) upon irradiation with pulses of different duration (see labels, all with $1 \mathrm{~J} / \mathrm{m}^{2}$ fluence, normal incidence, polarization across the ribbon width, and central photon energy $0.38 \mathrm{eV}$ tuned to the the transverse dipole ribbon plasmon). We show time-domain TB+RPA simulations obtained in the dyamical model with $\hbar \gamma^{T}=20 \mathrm{meV}, \hbar \gamma^{\mathrm{ph}}=5 \mathrm{meV}$. (f-k) Spectral decomposition of the dipoles presented in (b) (dashed curves) compared with those obtained in the static model (solid curves, taking $\hbar \gamma^{0}=25 \mathrm{meV}$ ). The green dotted curves show the incident light spectra for reference. Adapted with permission from Ref. ${ }^{52}$ Copyright 2018 Optical Society of America. 

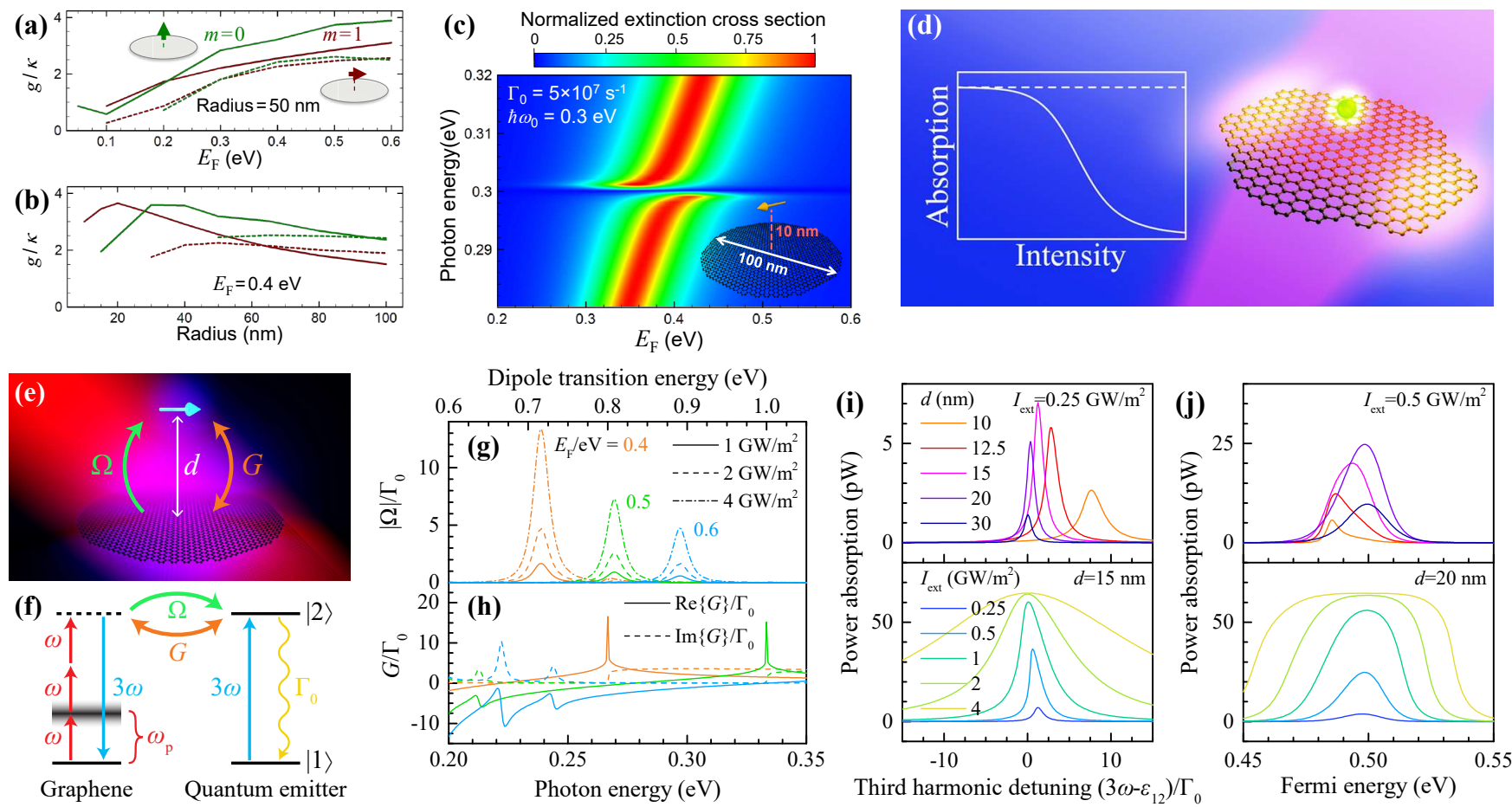

Figure 6: Interaction with quantum emitters (QEs). (a-c) Strong coupling and vacuum Rabi splitting in graphene nanodisks: in $(a, b)$ we show the Fermi-energy and size dependence of the strong coupling parameter $g / \kappa$ (QE-plasmon coupling rate $g$, plasmon decay rate $\kappa$ ) for a $\mathrm{QE}$ $\left(\Gamma_{0}=5 \times 10^{7} \mathrm{~s}^{-1}\right.$ natural decay rate) placed $10 \mathrm{~nm}$ above the center of a doped graphene disk of varying Fermi energy $E_{\mathrm{F}}$ and diameter $D$ (see horizontal axes and labels) when the emitter is tuned to the first (solid curves) or second (dashed curves) plasmon modes with either $m=0$ (green curves, QE out-of-plane polarization) or $m=1$ (red curves, QE polarization parallel to the graphene) azimuthal symmetries (photon energies scaling as $\propto \sqrt{E_{\mathrm{F}} / D}$ and having values $0.28 \mathrm{eV}$ and $0.16 \mathrm{eV}$, respectively, at $E_{\mathrm{F}}=0.4 \mathrm{eV}$ and $D=100 \mathrm{~nm}$ ); in (c) we show the extinction cross section (normal incidence, polarization in the graphene plane) revealing a clear vacuum Rabi splitting in the same QE-disk system (for $D=100 \mathrm{~nm}$ ) when plotted as a function of Fermi and photon energies with the emitter resonance at $0.3 \mathrm{eV}$ and its polarization parallel to the disk. Adapted with permission from Ref. ${ }^{8}$ Copyright 2011 American Chemical Society. (d) Illustration of strong-coupling regime: a QE can introduce a significant plasmon-plasmon interaction, giving rise to plasmon blockade associated with strongly nonlinear absorption cross sections and modified statistics of the bosonic plasmon mode. Adapted with permission from Ref. ${ }^{93}$ Copyright 2012 American Chemical Society. (e-j) Nonlinear QE-graphene plasmon coupling: in (e) we consider incident light (red beam, frequency $\omega$ tuned to the disk dipolar plasmon frequency $\omega_{\mathrm{p}}$ ) interacting with a graphene nanodisk to generate a nonlinear near-field (blue field, frequency $3 \omega$ ), which in turn couples to the QE (coupling Rabi frequency $\Omega$, plasmon-mediated self-interaction $G$ ) placed above the disk center; in (f) we show the energy-level diagram of the graphene-QE hybrid; in $(\mathrm{g}, \mathrm{h})$ we show calculations of $\Omega$ and $G$ for a $40 \mathrm{~nm}$ disk and a $20 \mathrm{~nm}$ disk-QE separation with normally incident $c w$ light of different intensities and various disk doping levels (see labels); in (i) we show the absorbed power of the hybrid system with $0.5 \mathrm{eV}$ doping (yielding a $\hbar \omega_{\mathrm{p}} \approx 0.27 \mathrm{eV}$ plasmon) as a function of light detuning from the QE resonance $\varepsilon_{12}=3 \omega_{\mathrm{p}}$ for different QE-disk separations $d$ and light intensities (see labels); in $(\mathrm{j})$ we show the dependence of absorption on doping level at the resonance condition $\omega=\varepsilon_{12} / 3$, using the same color code as in (i). Adapted with permission from Ref. ${ }^{53}$ Copyright 2018 American Physical Society. 

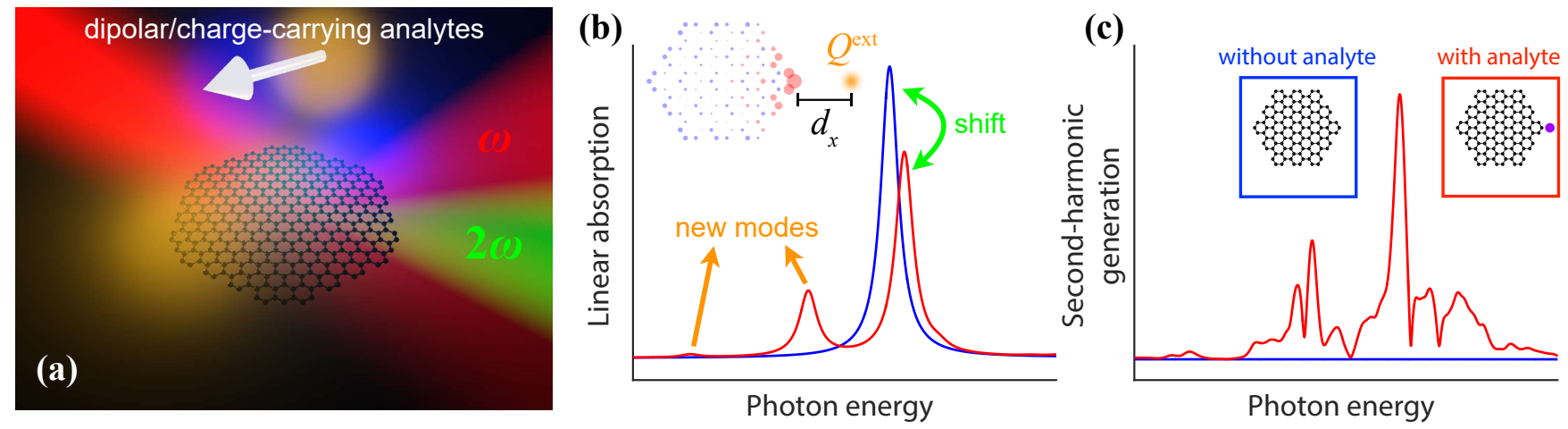

Figure 7: Nonlinear optical sensing with nanographenes. (a) We show a graphene nanoisland interacting with a charged or polar analyte, which influences the linear and nonlinear response of the former. (b) Changes produced in the electronic structure of the nanographene by the presence of, for example, a charged molecule can give rise to modifications in the linear response (e.g., plasmon shifts). (c) In graphene nanohexagons, the molecule can break the centrosymmetry of the structure, therefore enabling SHG, the detection of which can also be used to reveal the presence of the analyte. Adapted with permission from Ref. ${ }^{47}$ Copyright 2016 Americal Physical Society. 
(a)

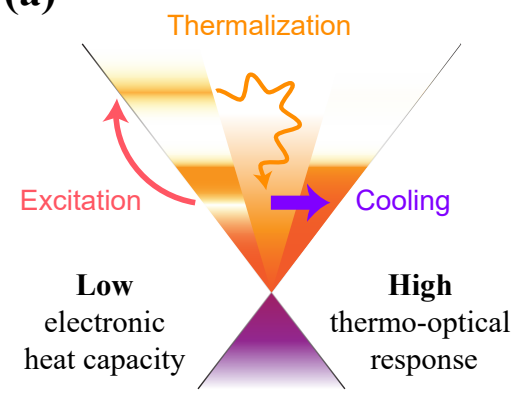

(d)

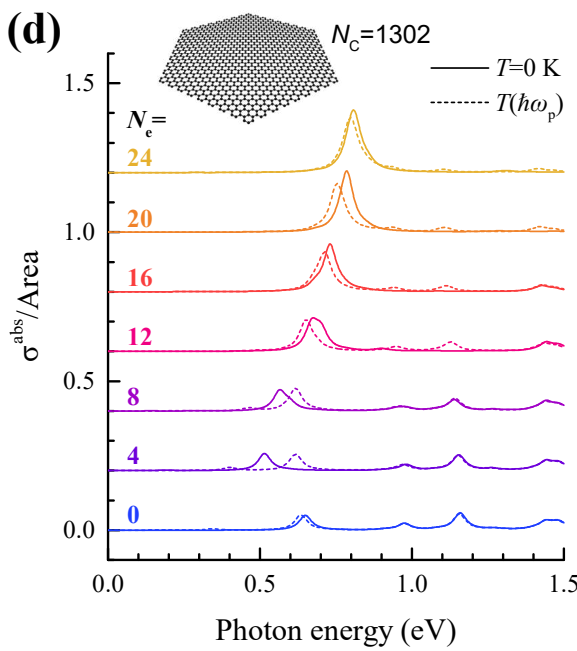

(b)
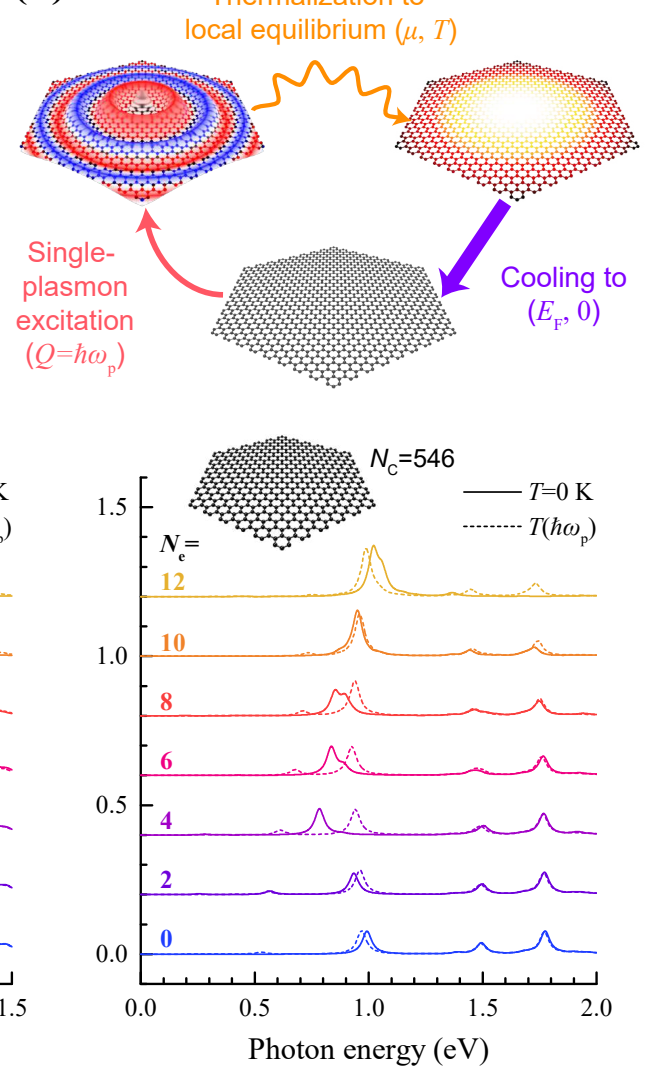

(c)
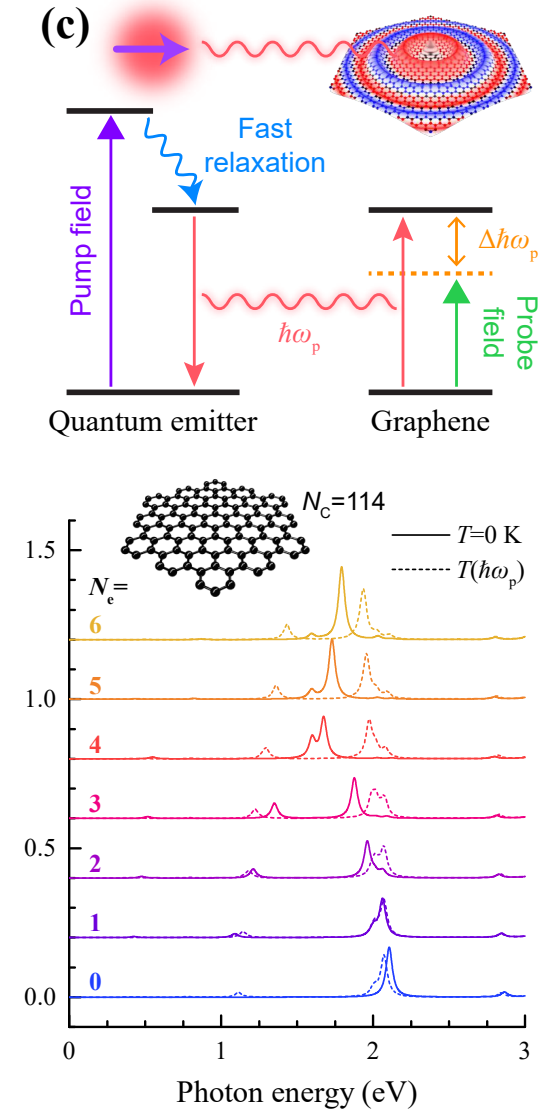

Figure 8: Single-photon thermal-optical switch. (a) Few hot carriers are sufficient to produce a high elevation of the electron temperature in graphene, giving rise to large changes in the optical response. (b) Sketch showing an unperturbed graphene nanohexagon (lower image), excited by a single-plasmon quantum of energy $\hbar \omega_{\mathrm{p}}$ (left image), which decays in tens of femtoseconds to produce electronic heating (temperature $T$ ) and a change in chemical potential $\mu$ (right image), followed by slow decay ( $\sim 1 \mathrm{ps}$ ) back to the unperturbed state. (c) Proposed scheme for single-plasmon thermooptical switching: a 3-level QE is optically pumped to a high-energy level, which decays fast to an intermediate state; the latter is tuned to the plasmon of a neighboring graphene island, thus causing the transfer of one-plasmon energy to the latter; a weak optical field then probes the change in the optical response of the graphene produced by thermo-optical plasmon energy shifting. (d) TB+RPA simulations of the absorption spectra of graphene armchaired nanohexagons consisting of $\mathrm{N}$ atoms (see upper insets) and doped with integral numbers of electrons $N_{\mathrm{e}}$ (see color-coordinated labels) before (solid curves) and after (dashed curves) single-plasmon absorption; smaller structures present larger single-plasmon tunability. Adapted with permission from Ref. ${ }^{85}$ Copyright 2019 American Chemical Society. 